\title{
Novel Quenched Disorder Fixed Point in a Two-Temperature Lattice Gas
}

\author{
B. Schmittmann and C. A. Laberge \\ Center for Stochastic Processes in Science and Engineering \\ and Department of Physics, Virginia Tech, Blacksburg, VA 24061-0435
}

(December 9, 1996)

\begin{abstract}
We investigate the effects of quenched randomness on the universal properties of a two-temperature lattice gas. The disorder modifies the dynamical transition rates of the system in an anisotropic fashion, giving rise to a new fixed point. We determine the associated scaling form of the structure factor, quoting critical exponents to two-loop order in an expansion around the upper critical dimension $d_{c}=7$. The close relationship with another quenched disorder fixed point, discovered recently in this model, is discussed.
\end{abstract}

PACS numbers: 64.60.Cn, 05.70.Fh, 82.20.Mj 
The statistical mechanics of non-equilibrium phenomena remains poorly understood, in spite of the ubiquity of such systems in nature. As a first inroad towards the full complexity of such systems, simple model systems driven into non-equilibrium steady states have attracted much interest over the past decade [1]. To prevent the systems from settling into the usual Boltzmann equilibrium steady state, a uniform current (of particles or energy) is maintained, e.g., by imposing a uniform drive, or by coupling the system to two reservoirs at different temperature or chemical potential. The prototype model is an Ising lattice gas, in the presence of a uniform "electric" field acting on the "charged" particles [2]. Motivated by the physics of fast ionic conductors [3], the usual Ising energetics is modified by the field which enhances (suppresses) the rate for nearest-neighbor particle-hole exchanges along (against) a specific lattice axis. The total number of particles is conserved, and periodic boundary conditions are imposed to ensure a non-vanishing global current. The resulting steady state distribution is non-Hamiltonian and violates detailed balance. In spite of its simplicity, this model, and many of its variants, exhibit surprising features like anomalous correlations at all temperatures and different types of non-equilibrium universal behavior [1].

In the framework of renormalization group ( $R G$ ) studies of these models, the universality class of order-disorder transitions is determined by the symmetries of the drive and the local order parameter alone, provided the dynamics is short-ranged in space and time. For equilibrium systems it is well known that spatial or temporal nonlocalities in the Hamiltonian, such as sufficiently long-ranged interactions or quenched impurities, can have profound consequences for critical behavior [4,5]. On the other hand, non-local terms appearing only in the dynamics but not in the Hamiltonian have no effect on static universal properties provided detailed balance holds. In contrast, if detailed balance is broken, the steady state is determined by both the local energetics and the dynamical rules, and nonlocalities in either are expected to play an important role.

So far, only a few studies have addressed this issue. The effects of long-ranged random spin exchanges, added to the non-conserved Glauber dynamics of the standard Ising model, have been investigated in some detail [6]. The resulting steady state, while Hamiltonian, is 
characterized by effective long-range interactions between spins. Other studies have focused on the effect of quenched disorder in the uniformly driven Ising lattice gas [7],8]. Here, due to the presence of a global current, a subtle complication arises when attempting to compare field theoretic results [7] with Monte Carlo data [8]. The simulations are performed on a finite lattice with periodic boundary conditions, so that particles typically see a given disorder configuration repeatedly in the course of thermal averaging, thus inducing effective correlations in the disorder average. In contrast, the analytic work rests on the assumption of uncorrelated randomness.

Motivated by an interest in deepening our understanding of quenched disorder in driven lattice gases, while recognizing the need to establish a clear correspondence between Monte Carlo data and field theory results, a non-equilibrium lattice gas without global current was studied in [9]. In the absence of quenched disorder (henceforth called the pure case), the field theory for this system captures the long-wavelength, long-time behavior of two different microscopic models: a randomly driven Ising model [10] and a two-temperature lattice gas [11]. Adding a quenched contribution to the drive in the randomly driven system, the authors showed that the field theory of the pure model is significantly modified, giving rise to a novel fixed point. The associated universal scaling behavior was investigated to two-loop order and found to be distinct from both the pure and the non-driven case.

In this Letter, we continue the analysis of quenched disorder in this system. Modelling the disorder as a quenched random bias acting along longitudinal or transverse bonds, we show that these two scenarios give rise to two distinct fixed points. The former of these, subsequently referred to as the "longitudinal" fixed point, was considered in 9]. Here, we investigate the other ("transverse") fixed point. We begin by summarizing the relevant properties of the pure system, followed by the introduction of quenched randomness. Next, we discuss the resulting effective Langevin equations for the longitudinal and transverse fixed points. The universal scaling behavior associated with the latter is then analyzed, using power counting techniques confirmed by an explicit $\epsilon$-expansion about the upper critical dimension of the theory. Explicit results for critical exponents are computed to two-loop 
order. We conclude with some comments.

In the absence of frozen disorder, our field theory describes the coarse-grained properties of two different microscopic models. The first, a randomly driven system [10], corresponds to a (half-filled) lattice gas of particles (up-spins) and holes (down-spins) on a fully periodic lattice, interacting through an attractive nearest-neighbor Ising coupling and coupled to a heat bath. As in the uniformly driven system, particle-hole exchanges along a specific lattice axis are biased by a driving field. Here, however, the amplitude $E$ of the drive switches sign and magnitude randomly in space and time, i.e., a new value of $E$ is culled from a symmetric distribution, $p(E)$, for each pair exchange along the field direction. Exchanges along the transverse direction are controlled by energetics alone. The symmetry of $p(E)$ ensures that there is no global current; otherwise its detailed form is not important for critical behavior. In its other version, a two-temperature model, we again consider a half-filled Ising lattice gas with spin-exchange dynamics. The drive, however, is now replaced by a second heat bath, such that spin pairs forming a "longitudinal" bond exchange according to a temperature $T_{\|}$while "transverse" bonds are controlled by a different temperature $T_{\perp}<T_{\|}$[11]. Both microscopic models obey the same set of global symmetries, namely: translation invariance along the longitudinal and the transverse directions, as well as rotation invariance in the $(d-$ 1)-dimensional transverse subspace. Moreover, both local order parameters are scalar and exhibit the usual up-down symmetry of the Ising model. The other common characteristic is that, as the (transverse) temperature is lowered, long-wavelength fluctuations with purely transverse wave vector become soft first, indicating that criticality sets in when the transverse diffusion coefficient vanishes while the longitudinal one is still positive. A Langevin equation for the local magnetization $\phi(x, t)$, consistent with these features, is easily postulated, or derived from its counterpart for the uniformly driven system by averaging with weight $p(E)$ [10]:

$$
\lambda^{-1} \partial_{t} \phi=\left(\tau_{\perp}-\nabla^{2}\right) \nabla^{2} \phi+\tau_{\|} \partial^{2} \phi+\frac{g}{3 !} \nabla^{2} \phi^{3}-\partial j_{\|}-\nabla j_{\perp} .
$$

Here $\partial(\nabla)$ denotes the gradients in the parallel (transverse) directions. $j_{\|}$and $j_{\perp}$ are 
Gaussian white noise terms which are distributed according to $\exp \left\{-\int d^{d} x d t\left[j_{\|}{ }^{2} / 4 n_{\|}+\right.\right.$ $\left.\left.j_{\perp}{ }^{2} / 4 n_{\perp}\right]\right\}$ and model the effect of thermal noise after coarse-graining. The parallel and transverse diffusion coefficients are denoted by $\tau_{\|}$and $\tau_{\perp}$. Criticality is associated with $\tau_{\perp} \rightarrow 0$ while $\tau_{\|}>0$. Since $\tau_{\|}$does not vanish, $\tau_{\|} \partial^{2} \phi$ is the only relevant term involving $\partial^{2}$. Otherwise, all coefficients here are functions of the microscopic control parameters but their explicit form is not required. Standard field theory methods [12] can be employed to compute the universal scaling behavior of the theory [10], in an expansion around the upper critical dimension $d_{c}=3$. High precision Monte Carlo data for the two-temperature model [13] are consistent with the field theory predictions, confirming that the two models do indeed belong to the same universality class.

Next, we introduce frozen disorder into the microscopic dynamics of the system, either in its random drive or two-temperature version, by adding a quenched random bias $\Delta_{\alpha}(x)$ to the exchange rate of a particle-hole pair located on a bond in the $\alpha$-direction, $\alpha=\|, \perp$. This type of randomness is easily implemented in a Monte Carlo simulation, since it can be considered as a time-independent local drive resident on each bond. In the Langevin equation (1), it leads to an extra term, $\vec{\nabla} \cdot \vec{J}$, on the right hand side, with the Ohmic current $J_{\alpha}$ given by $J_{\alpha}=c(\phi) \Delta_{\alpha}(x)$, in analogy to the uniformly driven case [1]. Here, $\vec{\nabla}$ is the full $d$-dimensional gradient, and $c(\phi) \propto 1+O\left(\phi^{2}\right)$ is the (density-dependent) conductivity. This form of $J_{\alpha}$ is also easily derived from a microscopic hopping model, upon taking a naive continuum limit and neglecting higher order derivatives. These, as well as the $O\left(\phi^{2}\right)$ correction to the conductivity, can be shown to be irrelevant in the RG sense. The $\Delta_{\alpha}(x)$ are taken to be Gaussian distributed with zero mean, $\left\langle\Delta_{\alpha}(x)>=0\right.$ so that no global current is induced, and $<\Delta_{\alpha}(x) \Delta_{\beta}\left(x^{\prime}\right)>=2 \sigma_{\alpha} \delta_{\alpha \beta} \delta\left(x-x^{\prime}\right)$. The positive parameters $\sigma_{\perp}$ and $\sigma_{\|}$control the strength of the randomness. It is now apparent that the leading modification of (1), apart from trivial redefinitions of its coefficients, is a quenched random current, $-\partial \Delta_{\|}-\nabla \Delta_{\perp}$, on the right hand side. To proceed, it is most convenient to introduce a Martin-Siggia-Rose field $\tilde{\phi}(x, t)$ [14], and to recast the Langevin equation as a dynamic functional [15]. In this form, the average over the $\Delta_{\alpha}(x)$ is easily performed, leading to an effective functional 


$$
\begin{aligned}
& \mathcal{J}[\phi, \tilde{\phi}]=\int d^{d} x \int d t \lambda \tilde{\phi}\left\{\lambda^{-1} \dot{\phi}-\left[\left(\tau_{\perp}-\nabla^{2}\right) \nabla^{2} \phi+\tau_{\|} \partial^{2} \phi+\frac{g}{3 !} \nabla^{2} \phi^{3}\right]\right\} \\
& +\int d^{d} x \int d t \lambda \int d t^{\prime} \lambda \tilde{\phi}(x, t)\left\{\left(n_{\|} \partial^{2}+n_{\perp} \nabla^{2}\right) \lambda^{-1} \delta\left(t-t^{\prime}\right)+\left(\sigma_{\|} \partial^{2}+\sigma_{\perp} \nabla^{2}\right)\right\} \tilde{\phi}\left(x, t^{\prime}\right) .
\end{aligned}
$$

We emphasize that both the frozen disorder and the thermal noise generate operators quadratic in $\tilde{\phi}$. In contrast to the thermal noise, however, the quenched random contribution to the Langevin current leads to an operator which is nonlocal in time. Correlation and response functions can now be computed as functional averages with weight exp $-\mathcal{J}[\phi, \tilde{\phi}]$.

The next step in the RG treatment of (2) is a dimensional analysis. Since $\tau_{\perp}$ vanishes first, parallel momenta scale as $k_{\|} \propto \mu^{2}$, on a characteristic scale $\mu \propto k_{\perp}$. Thus, strongly anisotropic scaling [1] is observed already at the tree level. As a consequence, the "transverse" coefficient $n_{\perp}$ is more relevant than its "longitudinal" counterpart, $n_{\|}$, which will therefore be dropped. Similarly, $\sigma_{\perp}$, if non-vanishing, is more relevant than $\sigma_{\|}$. A significant advantage of our particular implementation of disorder, as a bias acting on parallel or transverse bonds, now becomes apparent: it allows us to tune $\sigma_{\perp}$ or $\sigma_{\|}$separately to 0 . Here, we will choose $\sigma_{\perp} \neq 0$, in contrast to Ref. [9]. We will return to the consequences of either choice in the conclusions. Proceeding with the dimensional analysis, we can now neglect $\sigma_{\|}$as well. Requiring that the remaining terms in (2) be dimensionless, we find $\lambda t \propto \mu^{-4}$, so that the thermal noise becomes irrelevant and therefore negligeable compared to the quadratic term associated with the frozen disorder. Furthermore, choosing the scale of $\tilde{\phi}$ such that $\sigma_{\perp} \propto \mu^{0}$, we find $\phi \propto \mu^{(d-5) / 2}$ and $\tilde{\phi} \propto \mu^{(d+7) / 2}$, implying that $g \propto \mu^{7-d}$. The upper critical dimension follows as $d_{c}=7$, in contrast to the pure system where $d_{c}=3$. We also recall the result $d_{c}=5$ for a disordered system with $\sigma_{\perp}=0$ [9]. Thus, we may expect novel universal behavior for our theory. We should remark that such dimensional shifts have also been found in the uniformly driven system with frozen disorder [7].

We now consider the critical theory $\left(\tau_{\perp}=0\right)$ with insertions of $\lambda \tau_{\perp} \tilde{\phi} \nabla^{2} \phi$, to two-loop order in $\epsilon \equiv d_{c}-d$. A rescaling of $\tilde{\phi}$ allows us to set $\sigma_{\perp}=1$. The anisotropic forms of the bare correlation and response propagators are easily obtained from the Gaussian parts of $(2)$ : with $\Lambda(k) \equiv k_{\perp}^{2}\left(k_{\perp}^{2}+\tau_{\perp}\right)+\tau_{\|} k_{\|}^{2}$, we find $G_{o}(k, \omega)=[i \omega+\lambda \Lambda(k)]^{-1}$ for the 
response propagator, and $S_{o}(k, \omega)=4 \pi \lambda^{2} \delta(\omega)|i \omega+\lambda \Lambda(k)|^{-2}$ for the correlator. The latter, in particular, remains proportional to $\delta(\omega)$ at all orders, due to the presence of the timedelocalized noise in (2). A simple scale invariance of the theory, under $x_{\|} \rightarrow \gamma x_{\|}$at constant $x_{\perp}$ with arbitrary scale factor $\gamma$, identifies the effective dimensionless expansion parameter of the theory as $u \equiv \mu^{-\epsilon} \tau_{\|}^{-1 / 2} g$. Denoting one-particle irreducible vertex functions with $n$ $(\tilde{n})$ external $\phi-\left(\tilde{\phi}_{-}\right) \operatorname{legs}$ and $m$ insertions by $\Gamma_{\tilde{n} n}^{(m)}$, we find that only $\Gamma_{11}^{(0)}, \Gamma_{13}^{(0)}$, and $\Gamma_{11}^{(1)}$ are primitively divergent. Thus, only the quantities $\phi, u$, and $\tau_{\perp}$ must be renormalized to render the theory finite. The coupling constant $u$ flows towards an infrared stable fixed point $u^{*}$, while the renormalizations of $\phi$ and $\tau_{\perp}$ determine the only two independent critical exponents, $\eta$ and $\nu$. Following standard methods [12], the renormalization group equations at the fixed point, combined with dimensional analysis and scale invariance, predict the full asymptotic scaling behavior of the vertex functions $\Gamma_{\tilde{n} n}^{(m)}$. Instead of quoting the complete expressions here, we specialize to the steady-state structure factor, which is easily measured in simulations:

$$
S\left(k, t ; \tau_{\perp}\right)=\mu^{-6+\eta} S\left(k_{\|} / \mu^{1+\Delta}, k_{\perp} / \mu, t \mu^{z} ; \tau_{\perp} / \mu^{1 / \nu}\right)
$$

Some comments are in order here. First, the prefactor $\mu^{-6+\eta}$ may appear somewhat surprising, compared to the more familiar $\mu^{-2+\eta}$. However, its origin is easily traced back to the tree level result for the structure factor, $S_{o}(k, \omega)$, where the $\delta(\omega)$ is responsible for changing the familiar power of 2 to the more unusual 6 . Second, the strong anisotropy exponent $\Delta$ [1] and the dynamic exponent $z$ are related to the two independent exponents $\eta$ and $\nu$ via scaling laws: $\Delta=1-\eta / 4$ and $z=4-\eta / 2$. The latter differs from the familiar $z=4-\eta$ (for the conserved Ising model), again by virtue of the quenched disorder which modifies the usual relationships between the Wilson functions for $\phi, \tilde{\phi}$, and $\lambda$. The details of the two-loop calculation are too technical to be reported here, but the stability of the fixed point is established and explicit results for the critical exponents can be computed. We find $\eta \equiv \frac{88}{2187} \epsilon^{2}+O\left(\epsilon^{3}\right)$ and $\nu=\frac{1}{2}+\frac{\epsilon}{12}+\frac{\epsilon^{2}}{324}\left(\frac{1081}{81}+\ln \frac{4}{3}+6 \ln 4\right)+O\left(\epsilon^{3}\right)$, which shows clearly that our theory falls into a new universality class. 
Other scaling laws determine the remaining anisotropic exponents [1], such as the two different correlation length indices $\nu_{\perp}=\nu$ and $\nu_{\|}=\nu(1+\Delta)$, or the two different dynamic exponents $z_{\perp}=z$ and $z \|=z /(1+\Delta)$. Due to the anisotropy, care must also be taken when defining exponents that characterize the power-law decays of the critical structure factor, in real and momentum space. Simple scaling laws relate these indices to $\eta$ and $\Delta[\mathbb{1}]$. Finally, the order parameter exponent $\beta=\frac{1}{2} \nu(d-6+\eta+\Delta)$ follows from the scaling form of the equation of state [12].

In summary, we have analyzed the critical behavior of a two-temperature stochastic lattice gas with conserved dynamics, in the presence of a quenched random bias acting on bonds in both the longitudinal and transverse subspace. The bias generates a timedelocalized Langevin current which modifies the usual power counting so dramatically that the upper critical dimension is shifted to $d_{c}=7$. The RG analysis yields the asymptotic scaling forms of correlation and response functions; and critical exponents, associated with a new universality class, have been computed to $O\left(\epsilon^{2}\right)$ in an expansion about $d_{c}$. If, in contrast, the frozen disorder affects longitudinal bonds only, then $\sigma_{\perp}=0$ in Eqn (2). In this case, the dimensional analysis has to be revisited, resulting in $d_{c}=5$. Moreover, an additional relevant operator $-\kappa \int d^{d} x \int d t \int d t^{\prime} \lambda^{2} \tilde{\phi}(x, t) \nabla^{4} \tilde{\phi}\left(x, t^{\prime}\right)$ is generated under the RG and must be included in the dynamic functional. Considering the interplay between the two couplings $\sigma_{\|}$and $\kappa$, the former is found to be irrelevant while the latter gives rise to a new ("longitudinal") fixed point which is infrared stable near $d=5$ [9]. It is noteworthy [9] that this fixed point also captures the effect of quenched disorder resident in the nearest-neighbor hopping rates, modelled, e.g., by a Gaussian distribution of potential barriers between sites. Eqn (2), including the operators associated with $\sigma_{\|}, \sigma_{\perp}$ and $\kappa$ thus provides a unified field theory for several different types of quenched disorder. Work is in progress to clarify the intriguing connections between different microscopic implementations of such disorder and their surprising degree of universality [16]. 


\section{Acknowledgements:}

We wish to thank K.E. Bassler, V. Becker, H.K. Janssen, K. Oerding and R.K.P. Zia for many helpful discussions. We are particularly grateful to V. Becker and H.K. Janssen for communicating the results of Ref. [7] to us prior to publication. This work is supported in part by the National Science Foundation through DMR-9419393. 


\section{REFERENCES}

[1] B. Schmittmann and R.K.P. Zia, in: Phase Transitions and Critical Phenomena, Vol. 17, eds. C. Domb and J.L. Lebowitz (Academic, N.Y. 1995).

[2] S. Katz, J.L. Lebowitz, and H. Spohn, Phys. Rev. B28, 1655 (1983) and J. Stat. Phys. 34,497 (1984).

[3] J.W. Perram, The Physics of Superionic Conductors and Electrode Materials (Plenum, N.Y., 1983).

[4] See, e.g., A. Aharony, Phys. Lett. 44A, 313 (1973); M. Fisher, Rev. Mod. Phys. 46, 597 (1974). For a more recent discussion, see E. Luijten, H.W. Blöte and K. Binder, Phys. Rev. E54, 4626 (1996).

[5] see, e.g., R.B. Stinchcombe, in: Phase Transitions and Critical Phenomena, Vol. 7, eds. C. Domb and J.L. Lebowitz (Academic, N.Y. 1983).

[6] M. Droz, Z. Rácz and P. Tartaglia, Phys. Rev. A41, 6621 (1990) and Physica A177, 401 (1991); B. Bergersen and Z. Rácz, Phys Rev. Lett. 67, 3047 (1991); H.-J. Xu, B. Bergersen and Z. Rácz, Phys Rev. E47, 1520 (1993).

[7] V. Becker and H.K. Janssen, Europhys. Lett. 19, 13 (1992) and to be published.

[8] K.B. Lauritsen, 'Speciale' Thesis, Aarhus University, unpublished (1991); K.B. Lauritsen, and H.C. Fogedby, Phys. Rev. E47, 1563 (1992).

[9] B. Schmittmann and K.E. Bassler, Phys. Rev. Lett. 77, 3581 (1996).

[10] B. Schmittmann and R.K.P. Zia, Phys. Rev. Lett. 66, 357 (1991); B. Schmittmann, Europhys. Lett. 24, 109 (1993).

[11] P.L. Garrido, J.L. Lebowitz, C. Maes and H. Spohn, Phys. Rev. A42, 1954 (1990); C. Maes, J. Stat. Phys. 61, 667 (1990); Z. Cheng, P.L. Garrido, J.L. Lebowitz, and J.L. Vallès, Europhys. Lett. 14, 507 (1991). 
[12] see, e.g., J. Zinn-Justin, Quantum Field Theory and Critical Phenomena. (Oxford University Press, Oxford, 1989).

[13] E. Praestgaard, H. Larsen, and R.K.P. Zia, Europhys. Lett. 25, 447 (1994).

[14] P.C. Martin, E.D. Siggia and H.H. Rose, Phys. Rev. A8, 423 (1973).

[15] H.K. Janssen, Z. Phys. B23, 377 (1976); C. de Dominicis, J. Phys. (Paris) Colloq. 37, C247 (1976).

[16] B. Schmittmann and R.K.P. Zia, to be published. 